undertook an audit while doing our ear, nose, and throat (ENT) ST2 rotations for our general practice specialist training.

We were surprised by some of our findings. Of the 177 patients referred using the GP 2 WW pathway during a 7month period, $100 \%$ were meeting targets to be seen within 2 weeks, but only $6 \%(13 / 177)$ of these referrals were consequently diagnosed with head and neck cancer. In comparison to the incidence of cancer audited to be $23 \%^{1}$ for urology and $12.8 \%^{2}$ for breast 2-week wait referrals, ENT referrals appeared to have a much lower pick-up rate. In addition, of the $94 \%$ of the $2 \mathrm{WW}$ referrals which proved not to be cancer, we identified a sizable proportion that did not comply with the NICE $2 \mathrm{WW}$ referral guidelines.

While it is unavoidable that there will be a percentage of suspected cases that will have malignancy excluded by secondary care investigations, a large number of these 2WW ENT referrals may be given unnecessary priority over other general referrals thought to be less urgent, but which still affect a patient's quality of life.

Previously, other audits have shown a similar discrepancy with ENT referrals, and there have been multiple proposed reasons to explain this. For example, lack of knowledge of the $2 \mathrm{WW}$ referral criteria, or conversely, the poor predictive value of many of the $2 \mathrm{WW}$ referral criteria as symptoms suggestive of cancer. In addition, there has been suggestion of the inappropriate use of the $2 \mathrm{WW}$ referral system by primary care services. But perhaps there are significant contributing factors underlying all of these explanations - lack of clinical ENT experience, knowledge, and available diagnostics.

There is certainly some diagnostic difficulty with ENT malignancies relative to other types of cancer presenting in primary care. ENT symptoms of malignancy can be relatively nonspecific and there are a lack of diagnostic aids, such as tumour markers and radiological investigations, making a malignancy difficult to exclude without an early referral. This difficulty only highlights the importance of clinical acumen in ENT assessment and diagnosis in primary care.

Despite ENT conditions making up to $25-50 \%$ of all GP consultations, ${ }^{3}$ with many chronic ENT problems managed solely in primary care, practical knowledge and experience in dealing with ENT problems by GPs may have scope for improvement.

\section{Samantha Gardner,}

ST3 GP Vocational Training Scheme, Bath. E-mail: samanthagardner@doctors.org.uk

\section{Janaki Wijesekara,}

ST3 GP Vocational Training Scheme, Bath.

\section{REFERENCES}

1. Anup M, Desai KM. An audit of urology two-week wait referrals in a large teaching hospital in England. Annals of The Royal College of Surgeons of England 2009; 91(4): 310-312(3).

2. Potter S, Govindarajulu S, Shere M, et al. Referral patterns, cancer diagnoses, and waiting times after introduction of two week wait rule for breast cancer prospective cohort study. BMJ 2007; 335: 288.

3. Corbridge R, Steventon N. Oxford handbook of ENT and head and neck surgery. 2nd edn. Oxford: Oxford University Press, 2006.

DOI: 10.3399/bjgp09X472719

\section{Mozart in my practice}

In the August issue Dawn Brittain and Melvyn Jones investigated if music in the waiting room could be accepted by patients and staff. ${ }^{1}$ Discussing their research, they found debatable results, with a majority of patients in favour of music (mainly classical). There was no significant effect on patients' anxiety and health status.

I would like to highlight the relaxing side of music and analyse the effects of different kinds of music, depending on what we would like (or need) to achieve.

In this context, I thought about the use of music in my many years in practice and I realised that Mozart's music, and more specifically the piano concertos, are the best in my experience.

I find that Mozart's music can reduce the stress and tension in a busy and hectic general practice. I presented this theme in Zurich during an EQuiP meeting in October 1997 and again at the WONCA Congress in Palma de Mallorca in 1999.

For years I have used this music as light company in my waiting room and reiterate that the piano concertos are the best as they are smooth, without rapid changes of rhythm and sound, and with greater continuity.

The effects on patients are always very welcome; creating a relaxed environment and breaking any tension.

\section{Francesco Carelli}

Professor Family Medicine, University of Milan, EURACT Council Director of

Communications.

Email: carfra@tin.it

\section{REFERENCE}

1. Brittain D, Jones M. Music in the waiting room. $\mathrm{Br}$ Gen Pract 2009; 59: 613-614.

DOI: 10.3399/bjgp09X472728 